\title{
Supersonic gas injector for plasma fueling
}

V. A. Soukhanovskii, H. W. Kugel, R. Kaita, A. L. Roquemore, M. Bell, W. Blanchard, C. Bush, R. Gernhardt, G. Gettelfinger, T. Gray, R. Majeski, J. Menard, T. Provost, P. Sichta, R. Raman

October 3, 2005

The 21st IEEE/NPSS Symposium on Fusion Engineering (SOFE05)

Knoxville, TN, United States

September 26, 2005 through September 29, 2005 
This document was prepared as an account of work sponsored by an agency of the United States Government. Neither the United States Government nor the University of California nor any of their employees, makes any warranty, express or implied, or assumes any legal liability or responsibility for the accuracy, completeness, or usefulness of any information, apparatus, product, or process disclosed, or represents that its use would not infringe privately owned rights. Reference herein to any specific commercial product, process, or service by trade name, trademark, manufacturer, or otherwise, does not necessarily constitute or imply its endorsement, recommendation, or favoring by the United States Government or the University of California. The views and opinions of authors expressed herein do not necessarily state or reflect those of the United States Government or the University of California, and shall not be used for advertising or product endorsement purposes.

This work was performed under the auspices of the U.S. Department of Energy by University of California, Lawrence Livermore National Laboratory under Contract W-7405-Eng-48. 


\title{
Supersonic gas injector for plasma fueling
}

\author{
V. A. Soukhanovskii ${ }^{a}$, H. W. Kugel ${ }^{b}$, R. Kaita ${ }^{b}$, A. L. Roquemore ${ }^{b}$, M. Bell ${ }^{b}$, W. Blanchard ${ }^{b}$, C. $\operatorname{Bush}^{c}$, \\ R. Gernhardt ${ }^{b}$, G. Gettelfinger ${ }^{b}$, T. Gray ${ }^{b}$, R. Majeski ${ }^{b}$, J. Menard ${ }^{b}$, T. Provost ${ }^{b}$, P. Sichta $^{b}$, R. Raman $^{d}$ \\ ${ }^{a}$ Lawrence Livermore National Laboratory, Livermore, CA, USA \\ ${ }^{b}$ Princeton Plasma Physics Laboratory, Princeton, NJ, USA \\ ${ }^{c}$ Oak Ridge National Laboratory, Oak Ridge, TN, USA \\ ${ }^{d}$ University of Washington, Seattle, WA, USA
}

\begin{abstract}
A supersonic gas injector (SGI) has been developed for fueling and diagnostic applications on the National Spherical Torus Experiment (NSTX). It is comprised of a graphite converging-diverging Laval nozzle and a commercial piezoelectric gas valve mounted on a movable probe at a low field side midplane port location. Also mounted on the probe is a diagnostic package: a Langmuir probe, two thermocouples and five pickup coils for measuring toroidal, radial, vertical magnetic field components and magnetic fluctuations at the location of the SGI tip. The SGI flow rate is up to $4 \times 10^{21}$ particles/s, comparable to conventional NSTX gas injectors. The nozzle operates in a pulsed regime at room temperature and a reservoir gas pressure up to $0.33 \mathrm{MPa}$. The deuterium jet Mach number of about 4, and the divergence half-angle of $5^{\circ}-25^{\circ}$ have been measured in laboratory experiments simulating NSTX environment. In initial NSTX experiments reliable operation of the SGI and all mounted diagnostics at distances $1-20 \mathrm{~cm}$ from the plasma separatrix has been demonstrated. The SGI has been used for fueling of ohmic and 2-4 MW NBI heated L- and H-mode plasmas. Fueling efficiency in the range $0.1-0.3$ has been obtained from the plasma electron inventory analysis.
\end{abstract}

\section{INTRODUCTION}

Because of its technical simplicity, gas injection at the plasma edge is universally used for fueling of high-temperature plasmas in present day magnetically confined fusion (MCF) devices. Its well-known shortcomings are the low fueling efficiency, in the range $0.01-0.2$, and gas loading of invessel surfaces leading to uncontrolled fueling of plasma by out-gassing and recycling. The fueling efficiency is defined as $\eta=\left(d N_{i} / d t\right) \quad \Gamma_{g a s}^{-1}$, where $N_{i}$ is the confined particle inventory, and $\Gamma_{\text {gas }}$ is the gas injecton rate. Despite the shortcomings the gas injection technique is likely to complement other presently utilized fueling techniques - the high velocity cryogenic pellet injection and compact toroid injection - in future long-pulse experimental reactors, such as ITER [1]. A variation of the gas injection technique had been developed on the HL-1M tokamak [2], and later reproduced on several high-temperature MCF devices [3]-[6]: a high-pressure lowdivergence supersonic gas jet, also referred to as a supersonic molecular beam, is used for fueling the edge plasma. The gas jet is formed by a nozzle attached to a fast gas valve. A higher fueling efficiency of 0.3-0.6 and a reduced gas-wall interaction and therefore a lower wall saturation limit have been observed in L-mode plasma experiments in limiter tokamaks [7], [8]. In divertor tokamak experiments with the supersonic gas jet, a fueling efficiency of $0.1-0.3$ has been reported [6], [9].
A supersonic gas injector (SGI) has been developed on the National Spherical Torus Experiment (NSTX) [10], [11] to study the supersonic gas jet fueling and optimize long-pulse high-performance spherical tokamak plasmas. The SGI is a part of the NSTX particle control program which explores various wall conditioning techniques and active density control tools, such as cryopumping and low-recycling lithium surfaces. Plasma fueling is achieved in NSTX by injecting deuterium or helium through several piezoelectric valves with injection rates up to $10^{22}$ particles/s, and a fueling efficiency of about 0.1 [12], [13]. The NSTX SGI was designed to operate in a long pulse steady-state mode, injecting gas at a flow rate similar to conventional gas injectors, typically $N_{\text {pulse }} \simeq(0.1-0.5) \times N_{i}$. This distinguishes it from the other supersonic injectors [3]-[6] designed to inject gas in massive quantities $\Delta N_{\text {pulse }} \simeq(0.2-$ $0.9) \times N_{i}$ in milliseconds, leading to localized cooling of the plasma edge [14] or confinement degradation [6]. Experiments conducted in NSTX to date focused on characterization of the gas jet and its impact on the plasma edge.

\section{SUPERSONIC GAS INJECTOR DESIGN AND CHARACTERIZATION}

The SGI design incorporates several engineering and physics requirements: operational and vacuum autonomy, least interaction with NSTX machine operation and maintenance cycle, radial translation capability, gas throughput and diagnostic needs. For these reasons the SGI and several edge diagnostics are placed on a radially moving remotely controlled probe. The probe is mounted on a standard 4" diameter vacuum vessel port. The port is equipped with a machine vacuum interface valve and located at a distance $0.16 \mathrm{~m}$ above the midplane (Fig. 1). A ceramic break is used to isolate the probe electrically from the NSTX vacuum vessel potential. The SGI includes a nozzle, a valve and gas-handling hardware. The diagnostic package is comprised of a Langmuir probe, two thermocouples, and an Edge Magnetic Sensor (EMS) with five magnetic pick-up coils. The diagnostics and the valve with the nozzle are enclosed in a probe head covered by a protective shroud. The shroud is made of carbon fiber composite and ATJ graphite because of their low sputtering coefficient. All materials used in the SGI are ultra high vacuum compatible and bakeable to $125{ }^{\circ} \mathrm{C}$. The head is mounted on a Thermionics ZC-450 movable probe as shown in Fig. 2. The probe stroke is 24 ", with a step size 0.0005 " and a travel rate 


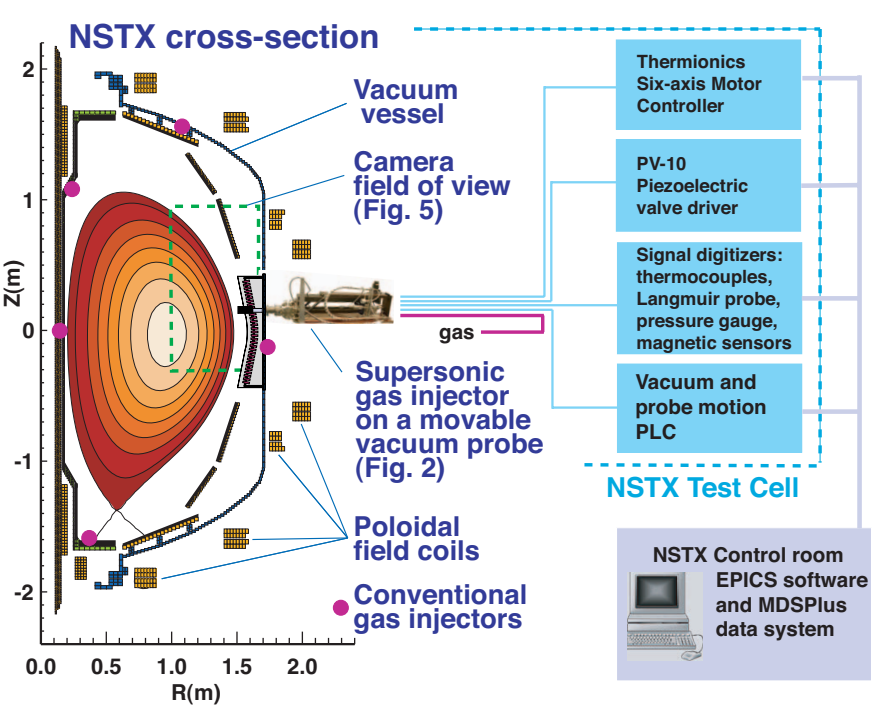

Fig. 1. Schematic of supersonic gas injector placement on NSTX and a block diagram of SGI control elements.

up to $15 \mathrm{in} / \mathrm{sec}$. A 0.5 " flexible gas line, a 0.25 " OD gas line and electric cables are routed along the stand and connected to the SGI through vacuum electrically isolated feed-throughs. A 0.5 " OD gas line and cables are mounted in vacuum inside the flexible probe bellows. The gas delivery lines are designed to handle flowing gas in a viscous regime with minimum boundary layer effects for a typical SGI deuterium pressure $P_{0} \leq 0.33 \mathrm{MPa}$. The gas delivery system includes a Sensotek Model TJE 5821-01 absolute pressure transducer for plenum pressure measurements before and after an injection and a fill isolation valve actuated by compressed air. The SGI gas line is connected to the NSTX Lower Dome and Inner Wall gas system [13] which uses standard gas bottles with a maximum regulator pressure of $0.33 \mathrm{MPa}$ imposed by hardware limits. The electric cables mounted inside the probe bellows are lowvoltage signal cables with an exception of a SGI piezoelectric valve driver cable. A Veeco PV-10 piezoelectric precision leak valve with a throat diameter 0.02 " is used in the SGI. The valve characteristic opening time is $1-2 \mathrm{~ms}$. Whereas the flow rate is proportional to the applied voltage in the range between 60 and $150 \mathrm{~V}$, we always operate the valve at $150 \mathrm{~V}$. The SGI plenum pressure variation is used to alter the flow rate. Several

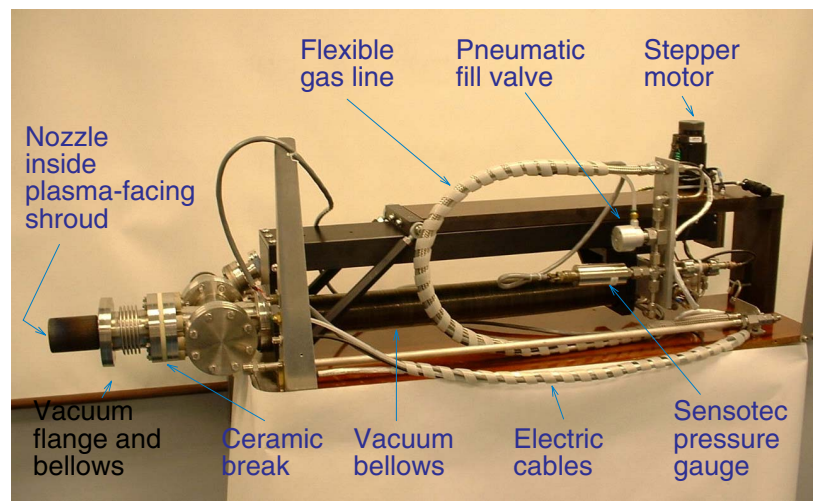

Fig. 2. Photograph of the SGI probe on a mounting stand. diagnostics are densely packed under the protective shroud around and behind the gas valve (Fig. 3). Integrated into the front surface of the shroud are a single Langmuir probe, a thermocouple, and two magnetic pick-up coils. The Langmuir probe is a flush-mounted type graphite tip probe 0.069 " in diameter. It is similar to the NSTX divertor tile probes used to determine the scrape-off layer (SOL) plasma potential, particle flux and with proper analysis, plasma temperature and density. The probe saturation current $I_{s a t}$ value in the tens of milliampers is obtained when the probe power supply voltage is swept between $-50 \mathrm{~V}$ and $50 \mathrm{~V}$. The thermocouples are used to monitor the shroud and the gas valve temperature during plasma operations. Two pick-up coils are mounted in slots on the front surface of the CFC shroud. Their spatial orientation enables measurements of the $B_{z}$ and $B_{t}$ components with a proper calibration. Three pick-up coils are mounted in a small shielded box behind the gas valve. Two of them are designed to measure radial and vertical magnetic field components $B_{r}, B_{z}$ while the third, Mirnov, coil is for edge magnetic fluctuation measurements.

The Thermionics probe is equipped with an encoder and a 6-axis stepper motor controller (SMC). EPICS software is used to communicate with the vacuum programmable logic control elements, such as the probe limit switches, vacuum and gas valves, and control the SMC remotely using its RS-232 port. The software is executed on an NSTX Engineering Network computer safeguarded by a firewall computer, and run from a terminal in the NSTX Control Room. The SGI head position has been calibrated in respect to the tokamak major radius $R$. The probe is usually parked at $R=1.98 \mathrm{~m}$ when unused, at $R=1.75 \mathrm{~m}$ in the glow discharge cleaning mode, and at $R=1.52-1.60 \mathrm{~m}$ (several $\mathrm{cm}$ from plasma) during plasma operations.

The SGI operation principle is based on diverse physics fields: gas-dynamics, compressible fluid mechanics, neutral gas transport, and magnetized plasma physics. An expansively cooled supersonic gas jet is obtained by expanding gas from a high pressure reservoir into vacuum through a nozzle. The jet penetrates through the plasma scrape-off layer perpendicular to the magnetic field, ionizes in the separatrix region and creates a localized high pressure plasma region. This plasmoid region expands along field lines, locally cooling

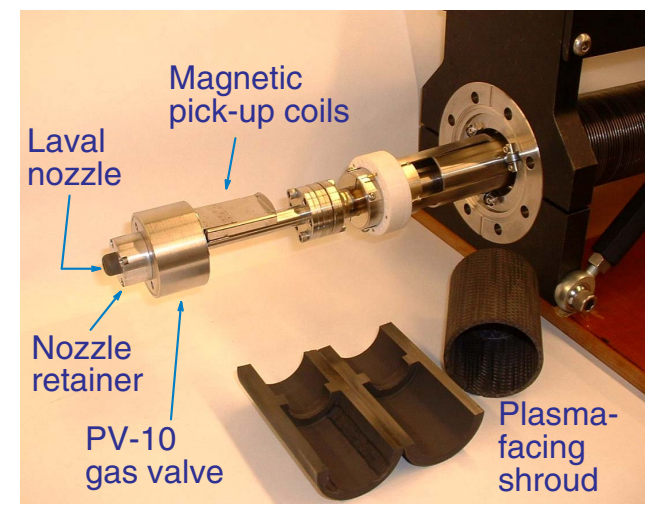

Fig. 3. Photograph of the SGI head assembly. 

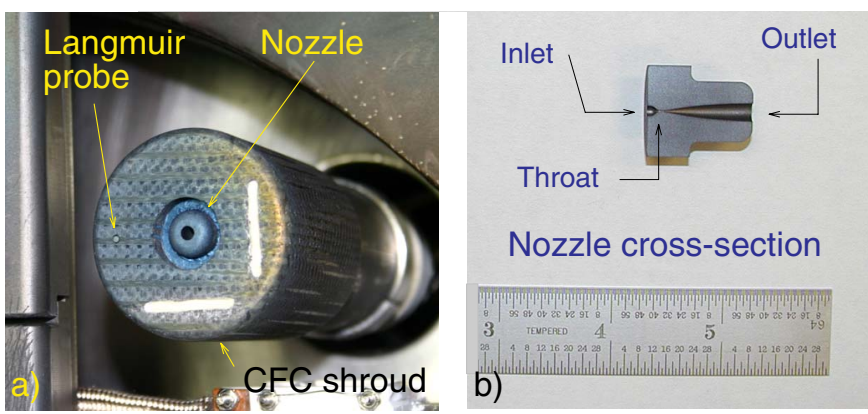

Fig. 4. Photographs of the SGI probe head inside the NSTX vacuum vessel (a) and the Laval nozzle (b). A flush-mount Langmuir probe tip and magnetic pick-up coil slots are visible on the SGI head.

and fueling the edge plasma. The SGI operates in a pulsed regime whereas any nozzle design relies on an established flow with steady-state parameters. The finite flow settle time limits the minimum SGI pulse length. The nozzle geometry determines supersonic jet properties, particularly the Mach number $M$ and the degree of clustering (and condensation) of gas molecules - two properties believed to be crucial for fueling applications. A low divergence high pressure jet is formed because of the Mach focusing of gas flow, while molecular clustering may increase the jet density by orders of magnitude. The jet terminal velocity $u_{\max }=\sqrt{\frac{2 \gamma}{\gamma-1} \frac{k T_{0}}{m}}$ is only a factor of $2-3$ greater than the thermal gas velocity $v_{t h}=\sqrt{3 k T_{0} / m}\left(\gamma\right.$ is the gas specific heat ratio, $T_{0}$ is the stagnation temperature). Four axisymmetric nozzle shapes are commonly used for making high quality supersonic gas jets: a converging nozzle (free jet), a converging-diverging Laval nozzle, a converging-diverging conical nozzle, and an aerospike (plug) nozzle [10]. In comparison with a simple converging nozzle, a shaped Laval nozzle produces a highly uniform flow with constant Mach number, temperature, and density - the conditions favorable for molecular condensation. A higher flow intensity can be obtained with a lower pressure ratio in a contoured nozzle avoiding problems associated with normal Mach disk shocks. The Laval nozzle shape must be

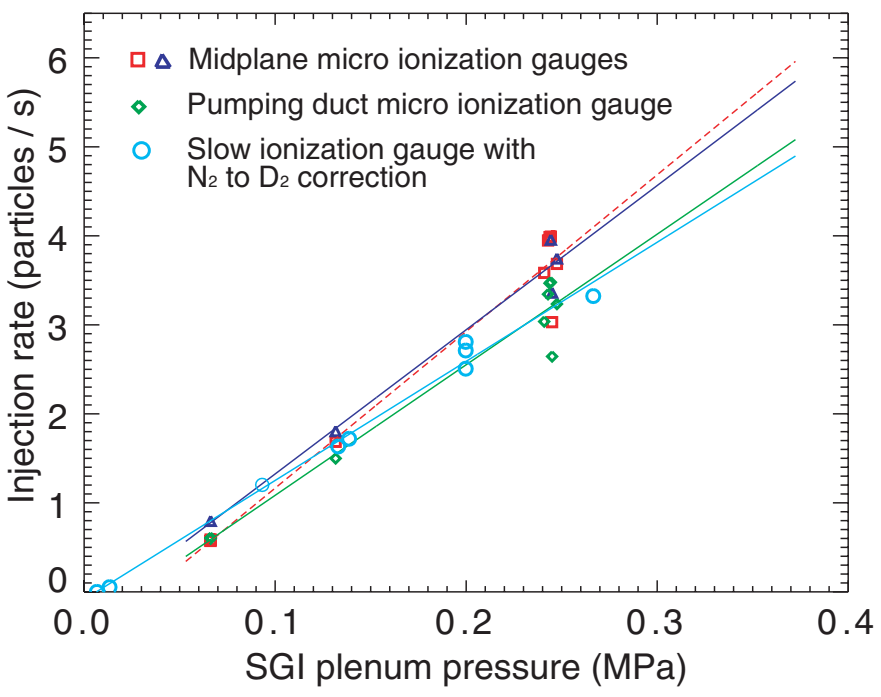

Fig. 5. Measured SGI rate as a function of SGI plenum pressure. Lines represent linear fit to the data.
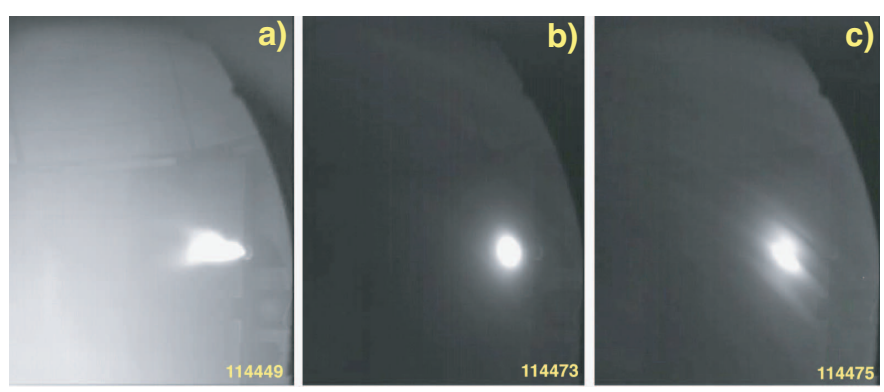

Fig. 6. Fast camera imaging of gas jet interaction with edge plasmas: (a) collapsing plasma with a wide $T_{e}=3 \mathrm{eV}, n_{e}=2-2.5 \times 10^{18} \mathrm{~m}^{-3}$ scrapeoff layer, (b) $6 \mathrm{MW}$ NBI-heated L-mode plasmas and (c) 4 MW NBI-heated H-mode plasmas.

properly calculated to optimize the isentropic flow core and minimize the thickness of the boundary layer. This is usually done using the method of characteristics or computational fluid dynamics methodology based on numerical solution of the Navier-Stokes equations. A converging-diverging de Laval geometry (Fig.4) is presently adopted in the NSTX SGI. The nozzle is mechanically machined from graphite with dimensional tolerance of $\pm 0.0025 "$ " The nozzle throat diameter is $d=0.01$ ", the inlet diameter is 0.087 ", and the exit diameter is 0.015 ". The nozzle is 0.92 " long. Nozzle performance in a pulsed regime has been evaluated in a simulated tokamak environment: the SGI injected gas pulses of $1-50 \mathrm{~ms}$ duration into a 50 liter vacuum tank with a background pressure $P_{b}=10^{-4}$ Torr, similar to the neutral pressures measured in NSTX [10], [11]. The deduced Mach number at the nozzle exit is about 4 for $\mathrm{D}_{2}$ or $\mathrm{H}_{2}$. The jet divergence half-angle is $\theta_{1 / 2} \simeq 6-12^{\circ}$. Using the isentropic relations between stagnation and static quantities the density at the jet exit is estimated to be $\rho \leq 10^{18} \mathrm{~cm}^{-3}$, and the temperature to be $T \geq 70 \mathrm{~K}$. The nozzle Reynolds number is $R e \simeq 6000$. These parameters indicate that the NSTX gas jet is not in the regime favorable for molecular cluster formation. Flow rates have been also calibrated on a laboratory stand and in-situ on NSTX using neutral pressure gauges (Fig. 5). The scatter of the measured flow rate is due to ionization gauge location proximity to the pump duct, calibration, and different plenum pressure differentials $\Delta P$ for gas pulse lengths 5 $300 \mathrm{~ms}$ used in the tests. The effective plenum volume of NSTX SGI is about $125 \mathrm{cc}$. Longer pulses lower the plenum pressure and reduce the effective flow rates up to $10 \%$ : for example, a $200 \mathrm{~ms}$ pulse causes a $5 \%$ decrease, whereas a $600 \mathrm{~ms}$ pulse causes a $15 \%$ decrease in the plenum pressure. A larger plenum will be installed in order to reduce this effect.

\section{Performance On NSTX}

Initial NSTX experiments focused on commissioning the SGI and the diagnostics, and studying the interaction of the supersonic gas jet with edge plasmas. Deuterium pulses of 50-500 ms duration have been injected in ohmic and NBIheated L- and H-mode plasmas. In ohmically heated plasmas the SGI head was brought as close as $1 \mathrm{~cm}$ to the plasma separatrix without any disruptive effects on the plasma or SGI head overheating. However, in NBI-heated plasmas the SGI head interacted with lost orbit energetic particles as soon as 
it cleared the radio-frequency antenna limiter. The resulting recycling and carbon flux from the SGI head caused a confinement degradation (H-L transitions) in $\mathrm{H}$-mode plasmas and disruptive MHD activity. In subsequent NBI-heated discharges the SGI was always positioned in the shadow of the limiter at a distance 6-12 $\mathrm{cm}$ from plasma separatrix. To monitor gas jet formation, evolution, and the SGI head and jet interaction with the SOL plasma fast camera imaging has been used. Shown in Fig. 6 are photographs of visible light from the supersonic gas jet interacting with different types of plasma taken by the Canadian Photonics camera with $1 \mathrm{~ms}$ frame rate and a line of sight $48^{\circ}$ with SGI axis. Gas jet divergence of $40-60^{\circ}$ degrees and the formation of a high-density region are inferred from the images confirming that the SGI operates in a supersonic regime. Shown in Fig. 7 are waveforms from a representative 2-4 MW NBI-heated H-mode plasma discharge fueled by the inner wall gas injector. An SGI pulse of $0.20 \mathrm{~s}$ duration is added starting at $0.18 \mathrm{~s}$ with a flow rate $\simeq 3.9 \times 10^{21}$ $\mathrm{s}^{-1}$. The SGI remains at a distance $8-13 \mathrm{~cm}$ from the plasma throughout the pulse duration. The SGI injection is clearly visible on the $D_{\alpha}$ emission waveform. Two inflections are seen on the line-integrated density $\bar{n}_{e}$ and total electron inventory $N_{e}$ time traces: the first one occurs at the inner wall gas injection onset time, and the second at the time of the SGI gas injection and an L-H transition onset. In order to calculate the SGI fueling efficiency $\left(d \Delta N_{e} / d t\right) \Gamma_{S G I}^{-1}$, fueling contributions from other gas injectors, recycling and impurities must be accounted for. Present analysis uses a difference between the total electron inventories $\Delta N_{e}$ of two consecutive discharges with and without an SGI pulse. The total electron inventory $N_{e}$ is obtained by volume-integrating the flux surface averaged Thomson scattering $n_{e}$ profiles for each time point. Fueling efficiency between 0.1 and 0.3 has been inferred in this manner, demonstrating high-density $\mathrm{H}$ mode plasma fueling with the supersonic gas jet. The SGI diagnostic package has been commissioned and used in several experiments to study the SGI performance and edge plasma characteristics. Shown in Fig. 7 are signal traces from the SGI probe diagnostics. Both thermocouples usually measure room temperatures throughout a plasma pulse. The Langmuir probe saturation current $I_{\text {sat }}$ time trace does not show any increase in SOL particle flux during the SGI pulse. The EMS has been used in the poloidal field (PF) only start-up experiments to monitor PF null formation, and during SGI pulses to monitor edge magnetic field perturbations, and edge MHD activity. The EMS signal traces shown in Fig. 7 use preliminary magnetic calibration factors.

\section{FUTURE WORK}

In summary, we have developed a pulsed supersonic gas injector for fueling and diagnostic applications on NSTX. Experiments to date have demonstrated the SGI fast time response, fueling efficiency in the range $0.1-0.3$, and compatibility with $\mathrm{H}$-mode plasmas. Future NSTX experiments will focus on development of high-performance plasma scenarios with supersonic gas jet fueling. Laboratory work will include testing of other nozzle geometries in an effort to optimize the SGI flow rate and gas jet divergence.

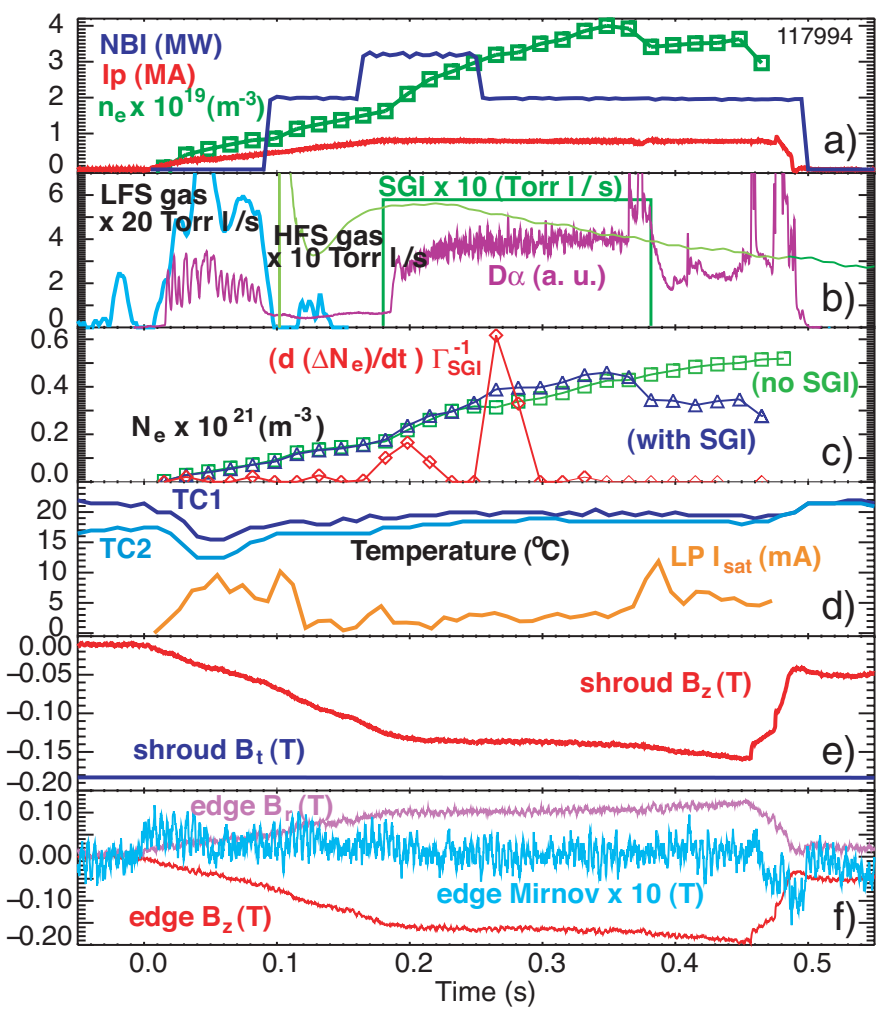

Fig. 7. Time traces of a representative SGI-fueled H-mode discharge. (a) Plasma current $I_{p}$, NBI power, line-averaged density $\bar{n}_{e}$, (b) Midplane $D_{\alpha}$ emission, gas flow rates for the low field side (LFS), high field side (HFS) injectors and SGI, (c) Total electron inventories for this shot and a shot without SGI, and the SGI fueling efficiency, (d) Shroud TC1 and gas valve TC2 thermocouple temperatures and Langmuir probe $I_{s a t}$, (e) Shroud coil sensor traces of $B_{t}$ and $B_{z}$, (f) edge coil sensor traces of $B_{r}, B_{z}$ and magnetic fluctuations (Mirnov).

\section{ACKNOWLEDGMENT}

We thank T. Czeizinger, J. Desandro, R. Feder, J. Gething, L. Guttadora, J. Kukon, D. Labrie, T. Holoman, and J. Winston for technical support. Prof. A. J. Smits and Dr S. Zaidi (Princeton University) are acknowledged for the nozzle design. NSTX team is acknowledged for plasma, NBI and diagnostic operations. This work is supported by U.S. DoE under Contracts No. W-7405-Eng-48 and DE-AC02-76CH03073.

\section{REFERENCES}

[1] ITER Physics Expert Group on Divertor, et. al., Nuc. Fusion, vol. 39, no. 12, p. 2391, 1999.

[2] L.Yao, et. al., Nuc. Fusion, vol. 38, no. 4, p. 631, 1998.

[3] X. Gao, et al., Nuc. Fusion, vol. 40, no. 11, p. 1875, 2000.

[4] J. Bucalossi, et al., in Proc. 29th Int Conf. on Fusion Energy. Lyon, France: IAEA, 2002, pp. EX/P4-04.

[5] J. Baldzuhn, et al., in Proc. 30th EPS Conf. on Contr. Fusion and Plasma Phys., vol. ECA 27A, St. Petersburg, 2003, pp. P-4.166pd.

[6] J. Bucalossi, et al., in Proc. 31st EPS Conf. on Plasma Physics, vol. ECA 28G, London, UK, 2004, pp. P-4.115.

[7] L. Yao, et al., Nuc. Fusion, vol. 44, no. 3, p. 420, 2004.

[8] B. Pegourie, et al., J. Nuc. Mater., vol. 313-316, p. 539, 2003.

[9] P. T. Lang, et al., Plasma Phys. Control. Fusion, vol. 47, p. 1495, 2005.

[10] V. A. Soukhanovskii, et al., Rev. Sci. Instrum., vol. 75, p. 4320, 2004.

[11] V. Soukhanovskii, et al., in Proc. 31st EPS Conf. on Plasma Physics, vol. ECA 28G, London, UK, 2004, pp. P-2.190.

[12] V. A. Soukhanovskii, et al., J. Nuc. Mater, vol. 313, p. 573, 2003.

[13] H. W. Kugel, et al., in Proc. 20th IEEE/NPSS Symposium on Fusion Eng. San Diego, CA: IEEE, October 2003.

[14] R. Panek, et al., J Nuc. Mater., vol. 337, no. 1-3, p. 530, 2004. 